\title{
Acoso escolar (bullying) como factor de riesgo de depresión y suicidio
}

\author{
Bullying as a risk factor for depression and suicide \\ Emilio Azúa Fuentesa,c, Pedro Rojas Carvallo ${ }^{\mathrm{a}, \mathrm{c}}$, Sergio Ruiz Poblete ${ }^{\mathrm{a}, \mathrm{b}}$
}

aDepartamento de Psiquiatría, Escuela de Medicina, Pontificia Universidad Católica de Chile, Santiago, Chile

baboratory for Brain-Machine Interfaces and Neuromodulation, Pontificia Universidad Católica de Chile, Santiago, Chile.

'Becado de Psiquiatría, Pontificia Universidad Católica de Chile, Santiago, Chile

Recibido: 3 de mayo de 2019; Aceptado: 22 de septiembre de 2019

\section{¿Qué se sabe del tema que trata este estudio?}

Sabemos que el bullying es un fenómeno altamente prevalente en población adolescente, y se ha asociado a intentos suicidas en esta población

\section{¿Qué aporta este estudio a lo ya conocido?}

El bullying es un factor de riesgo para ideación suicida en población cursando un episodio depresivo, y se asociaría directamente a ideación suicida en ciertos subgrupos. Se pone además de manifiesto la falta de estudios al respecto en Latinoamerica.

\section{Resumen}

Introducción: El acoso escolar (en inglés: bullying) corresponde a un abuso de poder sistematizado que se puede manifestar a través de distintos tipos de violencia, que puede ser física, verbal, exclusión social, entre otras. Dada la posible relación entre bullying y suicido, y su reciente repercusión mediática en nuestro país, es que se realiza esta revisión que explora la asociación entre bullying y desarrollo de depresión y/o suicidio. Objetivos: Revisar la literatura disponible y analizar la relación entre el acoso escolar (bullying) y el desarrollo de depresión y/o suicidio. Métodos: se realizó una búsqueda bibliográfica en las bases de datos PubMed, SciELO y LILACS con términos MeSH "bullying", "depression", "suicide", "acoso escolar", "depresión" y "suicidio", seleccionando para revisión 18 de 73 resultados. Resultados: Existe evidencia que establece relación entre exposición a bullying y desarrollo de depresión y suicidio en adolescentes, y enfatiza la importancia de la depresión como mediador entre el bullying y la conducta suicida. El cyberbullying es una nueva forma de expresión de este fenómeno, con evidencia en el desarrollo de depresión y suicidio, mayoritariamente en adolescentes mujeres. Es llamativa la escasez de estudios originales en Latinoamérica. Conclusiones: Pese a la heterogeneidad de los estudios, la evidencia refuerza la necesidad de mejorar los mecanismos de pesquisa y prevención de conductas de bullying entre adolescentes, con objetivo de disminuir la incidencia de depresión y suicidio en esta población.
Palabras clave:

suicidio;

bullying;

acoso escolar;

depresión;

cyberbullying 


\begin{abstract}
Introduction: Bullying is a systematized abuse of power that can be expressed through different types of violence such as physical, verbal, social exclusion, among others. Given the potential association between bullying and suicide, and its recent media repercussion in our country, this review explores the evidence that associates bullying and the development of depression and/or suicide. Objectives: To review the available literature and analyze the relationship between bullying and the development of depression and/or suicide. Methods: a bibliographic search was conducted in the PubMed, SciELO and LILACS databases with MeSH terms "bullying", "depression", "suicide", "acoso escolar", "depresión" and "suicidio", selecting 18 of 73 results for review. Results: There is evidence that establishes a relationship between exposure to bullying and the development of depression and suicide in adolescents, and emphasizes the importance of depression as a mediator between bullying and suicidal behavior. Cyberbullying is a new way of expression of this phenomenon, with evidence in the development of depression and suicide, mostly in female adolescents. The lack of original studies in Latin America is worrying. Conclusions: Despite the heterogeneity of the studies, the evidence reinforces the need to improve the mechanisms of research and prevention of bullying among adolescents, in order to reduce the incidence of depression and suicide in this population.
\end{abstract}

Keywords: bullying; depression; suicide; cyberbullying

\section{Introducción}

En los últimos dos años, la posible relación entre acoso escolar o bullying y suicidio infantil y adolescente se ha hecho de dominio masivo a través de una amplia cobertura mediática de gravísimos sucesos en nuestro país ${ }^{1,2}$. El bullying puede definirse como la exposición repetida en el tiempo a acciones negativas perpetradas por uno o más estudiantes, con los cuales se tiene una desventaja en términos de poder o fuerza ${ }^{3}$, manifestado de diferentes maneras en los ámbitos físicos, relacionales y verbales ${ }^{4}$. Del mismo modo, el cyberbullying presenta similares características que el bullying tradicional, con la diferencia de ser ejercido a través de un medio tecnológico. Por lo tanto, al analizar la asimetría de poder presente en el bullying, hay que considerar factores como diferencias en el manejo de tecnología y el anonimato del agresor ${ }^{3}$.

Según cifras de la Encuesta Nacional de Violencia en el Ámbito Escolar del año 2014, el 23,1\% de los estudiantes entre $7^{\circ}$ Básico y $4^{\circ}$ Medio declaran haber sido víctimas de agresiones verbales frecuentes, y el $20.2 \%$ declara haber sido víctimas de agresiones físicas frecuentes, cifras significativamente mayores que las obtenidas en las versiones previas de la encuesta ${ }^{5}$. Por otra parte, Chile cuenta con una tasa de suicidio de 10,7 personas por 100.000 habitantes entre los años 2014-2016 ${ }^{6}$. Esta cifra se ha ido incrementando, siendo Chile el país con el segundo aumento más importante en la tasa de suicidio entre los años 1990-2013 (después de Corea $)^{7}$, duplicando la tasa de mortalidad juvenil por suicidio del resto de Latinoamérica y el Caribe ${ }^{8}$.

De esta forma, se hace urgente y prioritario avanzar en la comprensión de la relación entre ambos fe- nómenos, i.e. bullying y suicidio. Este artículo tiene como objetivo revisar la literatura, tanto a nivel nacional como internacional, que objetive la relación entre bullying, depresión y la conducta suicida en población pediátrica. Se espera aportar a la comprensión de la relación entre estos factores, además de explorar el grado de conocimientos de este fenómeno en Latinoamérica y nuestro país.

\section{Métodos}

Se realizó una búsqueda bibliográfica en diciembre 2018, con los términos MeSH "bullying" "depression" y "suicide" (y sus equivalentes en español: acoso escolar, depresión, suicidio), en las bases de datos PubMed, LILACS y SciELO; se obtuvieron 64, 8 y 1 resultados respectivamente. De un total de 73 resultados, y luego de lectura de títulos y abstracts, se seleccionaron 18 artículos que cumplían los objetivos de la revisión, estando uno de ellos duplicado en las tres bases de datos. Se incluyeron trabajos en idioma español e inglés, y no se consideró límites en año de publicación de los artículos incluidos. No se incluyeron estudios de revisión sistemática.

\section{Resultados}

Se seleccionaron 18 artículos originales que cumplen con los criterios de búsqueda: 12 corresponden a muestras de Norteamérica, 2 de Europa, 2 de Asia, 1 de Oceanía y 1 de Sudamérica. No se encontraron artículos de Chile. Los artículos, muestras, diseño de estudio e instrumentos utilizados se resumen en la tabla 1 . 
Tabla 1. Detalle de origen y tamaño de muestra, diseño de estudio e instrumentos utilizados en cada estudio

\begin{tabular}{|c|c|c|c|c|}
\hline Autor & Muestra & Participantes & Diseño & Instrumentos \\
\hline $\begin{array}{l}\text { Bauman et al } \\
2013^{19}\end{array}$ & $\begin{array}{l}\text { Arizona, } \\
\text { Estados Unidos }\end{array}$ & $\begin{array}{l}\mathrm{n}=1.491 \text { estudiantes de } \\
\text { secundaria }\end{array}$ & Transversal & $\begin{array}{l}\text { Datos obtenidos del Arizona Youth Risk Behavior Surveillance } \\
\text { (YRBS) }\end{array}$ \\
\hline $\begin{array}{l}\text { Bonanno } \\
\text { et al } \\
2013^{26}\end{array}$ & $\begin{array}{l}\text { Columbia } \\
\text { Británica, } \\
\text { Canadá }\end{array}$ & $\begin{array}{l}n=399 \text { estudiantes de } \\
\text { secundaria }(M=14,2 \\
S D=0,91)\end{array}$ & Transversal & $\begin{array}{l}\text { Center for Epidemiological Studies-Depression Scale (CES-D) } \\
\text { Suicidal Ideation Questionnarie (SIQ-JR) }\end{array}$ \\
\hline $\begin{array}{l}\text { Espelage et al } \\
2013^{22}\end{array}$ & $\begin{array}{l}\text { Pueblo rural de } \\
\text { medio oeste, } \\
\text { Estados Unidos }\end{array}$ & $\begin{array}{l}n=661(10-13 \text { años; } \\
M=12,3)\end{array}$ & Transversal & $\begin{array}{l}18 \text {-item University of Illinois Aggression Scales } \\
2 \text { items del YouthSelf-report (Achenbach) para ideación } \\
\text { suicida } \\
13 \text { items del YouthSelf-report (Achenbach) para ansiedad y } \\
\text { depresión }\end{array}$ \\
\hline $\begin{array}{l}\text { Feng et al } \\
2016^{17}\end{array}$ & $\begin{array}{l}\text { Región de Salud } \\
\text { de Saskatoon, } \\
\text { Canadá }\end{array}$ & $\begin{array}{l}n=5.340 \text { estudiantes } \\
\text { entre } 9-14 \text { años }\end{array}$ & Transversal & Center for Epidemiological Studies-Depression Scale (CES-D) \\
\hline $\begin{array}{l}\text { Ford et al } \\
2017^{9}\end{array}$ & $\begin{array}{l}\text { Wave } 6 \text { de la } \\
\text { cohorte K del } \\
\text { estudio LSAC, } \\
\text { Australia }\end{array}$ & $\begin{array}{l}n=3.537 \text { adolescentes } \\
\text { entre } 14-15 \text { años }\end{array}$ & Transversal & $\begin{array}{l}11 \text { preguntas del School Climate Bullying Scale (Cornell, 2011) } \\
\text { Depresión medida con Short Mood and Feelings Question- } \\
\text { naire (MFQ) } \\
\text { Evaluación de suicidio del National Survey of Mental Health } \\
\text { and Wellbeing (Slade et al., 2009) }\end{array}$ \\
\hline $\begin{array}{l}\text { Hong et al } \\
2016^{18}\end{array}$ & Fujian, China & $\begin{array}{l}n=20.509 \text { estudiantes } \\
\text { (edad } 16,3 \pm 2 \text { años) }\end{array}$ & Transversal & $\begin{array}{l}6 \text { preguntas para determinar perpretadores de bullying } \\
6 \text { preguntas para determinar víctimas de bullying } \\
\text { Center for Epidemiological Studies-Depression Scale (CES-D) }\end{array}$ \\
\hline $\begin{array}{l}\text { Jones et al } \\
2014^{12}\end{array}$ & $\begin{array}{l}\text { Hospital de } \\
\text { niños de medio } \\
\text { oeste, Estados } \\
\text { Unidos }\end{array}$ & $\begin{array}{l}N=67 \text { adolescentes hospi- } \\
\text { talizados en salud mental } \\
(13-17 \text { años; } M=15,33 \\
S D=1,4)\end{array}$ & Transversal & $\begin{array}{l}\text { Bully Victimization Scale (BVS; Reynolds, 2003) } \\
\text { Children's Depression Inventory (CDI; Kovacs, 1992) } \\
\text { Inventory of Suicide Orientation-30 (ISO-30; King \& Kowal- } \\
\text { chuk, 1994) }\end{array}$ \\
\hline $\begin{array}{l}\text { Kerr et al } \\
2017^{13}\end{array}$ & $\begin{array}{l}\text { Oregon, } \\
\text { Estados Unidos }\end{array}$ & $\begin{array}{l}\mathrm{N}=206 \text { niños entre } 10-12 \\
\text { años. Seguimiento anual } \\
\text { hasta los } 32 \text { años, y luego } \\
\text { a los } 34 \text { años }\end{array}$ & Longitudinal & $\begin{array}{l}\text { Child Behavior Checklist (Achenbach \& Edelbrock, 1983) } \\
\text { Teacher Report Form (Achenbach \& Edelbrock, 1983) } \\
\text { Depression Self-Rating Scale (Birleson, 1981) } \\
\text { Entrevista diagnóstica (Shaffer et al, 1983) } \\
\text { Item de ideación suicida durante la última semana (Beck, } \\
\text { 1967) } \\
\text { Center for Epidemiological Studies-Depression Scale (CES-D) }\end{array}$ \\
\hline $\begin{array}{l}\text { King et al } \\
2013^{16}\end{array}$ & $\begin{array}{l}\text { Michigan, } \\
\text { Estados Unidos }\end{array}$ & $\begin{array}{l}N=433 \text { adolescentes } \\
\text { suicidas entre } 13 \text { y } 17 \\
\text { años, hospitalizados en } \\
\text { salud mental, edad media: } \\
15,6 \text { años }\end{array}$ & Longitudinal & $\begin{array}{l}\text { The Suicidal Ideation QuestionnairedJunior (SIQ-JR) } \\
\text { The Children's Depression Rating ScaledRevised (CDRS-R) } \\
\text { Seis ítems del The Youth Self Report (YSR) }\end{array}$ \\
\hline $\begin{array}{l}\text { Klomek et al } \\
2011^{14}\end{array}$ & $\begin{array}{l}\text { Nassau, Suffolk } \\
\text { y Westchester, } \\
\text { Nueva York, } \\
\text { Estados Unidos }\end{array}$ & $\begin{array}{l}\mathrm{n}=2.342 \text { estudiantes } \\
\text { entre } 13 \text { y } 18 \text { años, edad } \\
\text { media } 14,8 \text { años }\end{array}$ & Longitudinal & $\begin{array}{l}\text { Beck Depression Inventory (DBI-IA), Suicidal Ideation Ques- } \\
\text { tionnaire (SIQ-JR) } \\
\text { Siete Preguntas respecto de experiencias de bullying derivadas } \\
\text { del World Health Organization study on youth health. }\end{array}$ \\
\hline $\begin{array}{l}\text { Klomek et al } \\
2013^{21}\end{array}$ & $\begin{array}{l}\text { Nassau, Suffolk } \\
\text { y Westchester, } \\
\text { Nueva York, } \\
\text { Estados Unidos }\end{array}$ & $\begin{array}{l}\mathrm{n}=317 \text { adolescentes en- } \\
\text { tre } 13 \text { y } 18 \text { años con riesgo } \\
\text { psiquiátrico, obtenidos } \\
\text { de una cohorte de } 2.342 \\
\text { estudiantes }\end{array}$ & Longitudinal & $\begin{array}{l}\text { Beck Depression Inventory (BDI-IA) } \\
\text { Suicidal Ideation Questionnarie (SIQ-JR) } \\
\text { Siete Preguntas respecto de experiencias de bullying derivadas } \\
\text { del World Health Organization study on youth health. }\end{array}$ \\
\hline $\begin{array}{l}\text { Lardier et al } \\
2016^{11}\end{array}$ & $\begin{array}{l}\text { Nueva Jersey, } \\
\text { Estados Unidos }\end{array}$ & $\begin{array}{l}n=488 \text { adolescentes, } \\
\text { edad promedio } 18,81 \text { años }\end{array}$ & Transversal & $\begin{array}{l}\text { Preguntas derivadas del School Relationships Questionnaire } \\
\text { Preguntas derivadas del Child behaviour Checklist } \\
\text { Dos preguntas del Youth Risk Behavioural Surveillance } \\
\text { Survey }\end{array}$ \\
\hline $\begin{array}{l}\text { Le } \\
2017^{15}\end{array}$ & $\begin{array}{l}\text { Delta del Río } \\
\text { Rojo, Vietnam }\end{array}$ & $\begin{array}{l}n=1.424 \text { escolares } \\
\text { adolescentes. Edad media } \\
14,7 \text { años }\end{array}$ & Longitudinal & $\begin{array}{l}\text { Olweus Bully/Nictim Questionnaire } \\
\text { Center for Epidemiological Studies-Depression Scale (CES-D) } \\
\text { Tres items adaptados del American School Health Association }\end{array}$ \\
\hline $\begin{array}{l}\text { Lereya et al } \\
2015^{10}\end{array}$ & $\begin{array}{l}\text { Reino Unido. } \\
\text { Estados Unidos }\end{array}$ & $\begin{array}{l}\text { Cohorte ALSPAC } n=4.026 \\
\text { Cohorte GSMS } n=1.420\end{array}$ & Transversal & $\begin{array}{l}\text { Bullying and Friendship Interview Schedule } \\
\text { Child and Adolescent Psychiatric Assessment (CAPA) } \\
\text { Clinical Interview Schedule (CIS-R) } \\
\text { Young Adult Psychiatric Assessment (YAPA) }\end{array}$ \\
\hline
\end{tabular}


Tabla 1. Detalle de origen y tamaño de muestra, diseño de estudio e instrumentos utilizados en cada estudio (continuación)

\begin{tabular}{|c|c|c|c|c|}
\hline Autor & Muestra & Participantes & Diseño & Instrumentos \\
\hline $\begin{array}{l}\text { Meltzer et al } \\
2011^{20}\end{array}$ & Inglaterra & $\begin{array}{l}\mathrm{n}=7.461 \text { personas en- } \\
\text { cuestadas }\end{array}$ & Transversal & $\begin{array}{l}\text { Clinical Interview Schedule [the CIS-R] } \\
\text { Dos preguntas basadas en trabajos de Paykel et al y Salmons } \\
\text { and Harrington para evaluar suicidio. } \\
\text { Stressful Life Events }\end{array}$ \\
\hline $\begin{array}{l}\text { Sandoval-Ato } \\
\text { et al } \\
2017^{24}\end{array}$ & Piura, Perú & $\begin{array}{l}n=296 \text { adolescentes, } \\
\text { edad media } 14,4 \text { años } \\
\text { (rango } 13-16 \text { años) }\end{array}$ & Transversal & $\begin{array}{l}\text { Cuestionario de salud mental del Instituto Nacional de Salud } \\
\text { Mental del Perú } \\
\text { Escala de Plutchik } \\
\text { European Bullying Intervention Project Questionnaire, (EBIPQ) } \\
\text { European Cyberbullying Intervention Project Questionnaire, } \\
\text { (ECIPQ) } \\
\text { Escala de Depresión de Beck }\end{array}$ \\
\hline $\begin{array}{l}\text { Undheim } \\
2013^{23}\end{array}$ & $\begin{array}{l}\text { Dos condados } \\
\text { centrales, No- } \\
\text { ruega }\end{array}$ & $\begin{array}{l}n=2.464 \text { adolescentes, } \\
\text { edad media } 13,7 \text { años } \\
(12,5-15,7)\end{array}$ & Longitudinal & $\begin{array}{l}\text { Escala construida compuesta de } 6 \text { puntos en } 3 \text { ítems. } \\
4 \text { items del Youth self-report. } \\
\text { Mood and Feelings Questionnaire (MFQ) }\end{array}$ \\
\hline $\begin{array}{l}\text { Williams et al } \\
2017^{25}\end{array}$ & $\begin{array}{l}\text { Alabama, Esta- } \\
\text { dos Unidos }\end{array}$ & $\mathrm{n}=233$ estudiantes & Transversal & $\begin{array}{l}\text { Bully Survey (Swearer et al., 2010) } \\
\text { Una pregunta dicotomica par determinar cyberbullying. } \\
\text { Centers for Epidemiological Studies Depression Survey-10 } \\
\text { (CESD-10) } \\
\text { Una pregunta para evaluar ideación suicida. } \\
\text { Una pregunta para evaluar intento suicida. }\end{array}$ \\
\hline
\end{tabular}

\section{a) Relación entre bullying y depresión}

En 8 artículos se analizan relaciones estadísticas entre bullying y depresión, 4 de ellos son estudios transversales y 4 son longitudinales. Se estableció en ellos relación estadística entre exposición a bullying y síntomas depresivos en población pediátrica.

\section{Estudios transversales}

Ford y cols. ${ }^{9}$ mediante regresión lineal ajustada por confundentes determinaron una relación significativa entre bullying y síntomas depresivos en adolescentes, presentando el bullying físico un $\beta=1,6$, bullying relacional-verbal $\beta=4,2$ y ambas formas un $\beta=5,1$. Según el rol de participación en bullying se determinó que, respecto de los no involucrados, tanto las victimas, como los agresores y los agresores-victimas aumentan sus puntajes en las escalas de depresión.

Lereya y cols. ${ }^{10}$ en dos cohortes de niños realizaron una comparación mediante análisis de regresión logística binaria entre dos grupos, uno expuesto y otro no expuesto a bullying, determinando en ambas cohortes que el grupo expuesto presentó más riesgo de depresión (ALSPAC: $\mathrm{OR}=2,3, \mathrm{p}<0,001$; GSMS: $\mathrm{OR}=5,8$, $\mathrm{p}<0,001)$.

Lardier y cols. ${ }^{11}$ identificaron una correlación directa entre experiencia de bullying y depresión tanto para hombres como para mujeres $(\mathrm{OR}=0,276 \mathrm{y}$ $\mathrm{OR}=0,237$, ambos $\mathrm{p}<0,001)$. Sin embargo, al aplicar modelo de mediación múltiple, este efecto persistió solo en mujeres $(b=0,124, p<0,01)$.
En un grupo de adolescentes hospitalizados en salud mental, Jones y cols. ${ }^{12}$ demostraron mediante análisis de correlación bi-variada que el ser víctima de bullying se correlaciona con depresión $(r=0,36$, $\mathrm{p}<0,001)$.

\section{Estudios longitudinales}

Kerr y cols. ${ }^{13}$ en una cohorte de niños, no observaron que el hecho de estar involucrado en experiencias de bullying sea predictor de síntomas depresivos posteriores luego de ajustar por factores confundentes.

Klomek y cols. ${ }^{14}$ determinaron la presencia o ausencia de riesgo psiquiátrico en un grupo de adolescentes, riesgo determinado por la evaluación de niveles de suicidalidad, depresión o uso de sustancias. Además, en ambos grupos determinaron el involucramiento o no en conductas de bullying (ya sea como perpetrador, víctima o ambos). En ambos grupos (con y sin riesgo psiquiátrico) los involucrados en bullying presentaron promedios significativamente mayores en depresión $(\mathrm{t}=5,59, \mathrm{p}<0,001)$. En el seguimiento a 4 años, los estudiantes victimas de bullying reportaban mayor ideación suicida que los perpetradores de bullying $(\mathrm{t}=2,51, \mathrm{p}<0,05)$.

Le y cols. ${ }^{15}$ describieron que los adolescentes involucrados en bullying, ya sea como víctima o perpetradorvíctima, presentan más síntomas depresivos respecto a los no involucrados. Al aplicar análisis multivariado determinaron que quienes presentan altos niveles de victimización son más propensos a presentar síntomas 
depresivos ( $\beta=6,6, p<0,001)$, junto también a quienes siendo perpetradores-víctimas mantienen un alto nivel de agresión hacia otros ( $\beta=6,1$ y $\beta=6,0$ respectivamente, ambos $\mathrm{p}<0,001)$.

En un grupo de adolescentes suicidas hospitalizados, King y cols. ${ }^{16}$ evaluaron diferencias entre los perpetradores de bullying versus los no perpetradores, $\mathrm{y}$ determinaron que al inicio del estudio no presentan diferencia significativa en el puntaje de depresión (63,6 vs $60,7, p=0,12)$, y en el seguimiento a 12 meses, el descenso en los puntajes no varía significativamente.

\section{b) Relación entre bullying y suicidio}

En 14 artículos originales se estudió la relación entre bullying y suicidio (ya sea ideación, intento o ambos). Ocho estudios son estudios transversales y 6 son longitudinales, estableciendo en la mayoría de ellos relación entre exposición a bullying y riesgo suicida.

\section{Estudios transversales}

En los estudios transversales se observaron resultados son variados. En relación con ser víctima de $b u$ llying e ideación suicida todos los estudios encontraron algún grado de asociación, que varía entre $\mathrm{OR}=1,82^{17}$ y $\mathrm{OR}=3,4^{9}$. Un estudio de Hong y cols. ${ }^{18}$ encontró una asociación entre ser víctima de bullying e ideación suicida $(\mathrm{OR}=2.74, \mathrm{p}<0,001)$ y tras ajustar por depresión se observó que esta asociación persiste, pero en menor grado $(\mathrm{OR}=1,49, \mathrm{p}=0,018)$.

Respecto a la incidencia de intentos de suicidio y su relación con haber sido víctima de bullying hay información variada, e incluso contradictoria. Bauman y cols. ${ }^{19}$ concluyeron que, al ajustar por síntomas depresivos, no habría una relación significativa entre haber sido partícipe de bullying e intentos de suicidio a futuro. Por otro lado, Ford y cols. ${ }^{9}$ encontraron, mediante regresión de Poisson, una asociación entre haber sido víctima de bullying y presentar al menos un intento de suicidio durante la adolescencia $(P R R=2,3)$. Dicha asociación fue mayor en aquellos adolescentes que tienen antecedentes de haber estado involucrados como víctimas y también como agresores ( $\mathrm{PRR}=2,7)$. Estos resultados son complementarios a los encontrados por el grupo de Meltzer y cols. ${ }^{20}$, quienes demostraron una asociación entre haber sido víctima de bullying e intentos de suicidio en la adultez $(\mathrm{OR}=2.35, \mathrm{p}<0,001)$.

$\mathrm{Al}$ analizar diferencias por sexo, Lardier y cols. ${ }^{11}$ observaron que en adolescentes el bullying produciría por sí solo un aumento de la probabilidad de ideación suicida, y que esto afectaría distintamente a hombres (7\%) y a mujeres $(9 \%)$. Sin embargo, mediante un análisis de regresión logística multivariada este efecto dejó de ser significativo al incluir otras variables de riesgo como depresión, conflicto familiar, síntomas ansiosos y uso de sustancias.

\section{Estudios longitudinales}

En estudios longitudinales se describe una clara asociación entre haber participado como víctima y perpetrador de bullying, y futura ideación e intentos suicidas. El grupo de Le y cols..$^{15}$ encontró un aumento de ideación suicida tanto en víctimas, perpetradores y perpetrador-víctima. En los primeros 2 grupos el aumento de riesgo dependió del grado de involucramiento en bullying, mientras que el grupo de perpetradoresvíctimas presentó un aumento de riesgo en todos los niveles de involucramiento. En el análisis multivariado se observó un riesgo máximo en el grupo de mujeres perpetradores-víctimas con involucramiento establealto $(\mathrm{OR}=6,5, \mathrm{p}<0,001)$. Por otro lado, el grupo de Kerr y cols. ${ }^{13}$ estudió la relación entre la participación en bullying y riesgo de intentos de suicidio, encontrando una asociación significativa para el grupo de víctimas-agresores $(\mathrm{OR}=7,56, \mathrm{p}<0,01)$.

El grupo de King y cols. ${ }^{16}$ observó que a los 12 meses de seguimiento no hubo una asociación significativa entre la presencia de ideación suicida en adolescentes y haber estado involucrado en bullying. Sin embargo, el grupo de Klomek y cols. ${ }^{21}$ reportó que en adolescentes con riesgo suicida previo, el antecedente de haber participado de bullying como perpetrador aumentó la presencia de ideación suicida en el seguimiento a dos años (media $=8,5$ vs $13,9, \mathrm{p}<0,05)$.

\section{c) Relación entre bullying y suicidio mediado por depresión}

En 5 estudios transversales y 2 longitudinales se evaluó la relación entre bullying y suicidio mediado por depresión, encontrándose datos estadísticamente significativos para dicha mediación.

\section{Estudios transversales}

En el estudio transversal de Hong y cols. ${ }^{18}$, luego de confirmar una interacción significativa entre depresión y bullying, se demostró mediante modelos de regresión logística que los adolescentes con depresión presentaron mayor probabilidad de ideación suicida $(\mathrm{OR}=6,27, \mathrm{p}<0,001)$.

Jones y cols. ${ }^{12}$ realizaron un análisis de correlación bi-variada en un grupo de adolescentes, encontrando que se correlaciona el ser víctima de bullying con ideación suicida ( $\mathrm{r}=0,33, \mathrm{p}<0,001)$, mediado por el componente "autoestima negativa". En el estudio de Meltzer y cols. ${ }^{20}$, tras realizar un análisis de mediación, se demostró que la asociación entre bullying en la infancia y suicidio en la adultez persiste significativamente tras controlar por depresión $(\mathrm{t}=8,09, \mathrm{p}<0,001)$.

Por otro lado, el grupo de Lardier y cols. ${ }^{11}$ reportó que el bullying no es un indicador significativo de ideación suicida, sugiriendo que otros factores son mediadores entre ambos. Se observó que en presencia de 
síntomas depresivos el bullying aumentó la probabilidad de riesgo suicida en un $52 \%$ en hombres y $37 \%$ en mujeres. En el desarrollo de ideación suicida a partir de experiencias de bullying, la depresión fue identificada como mediador sólo en mujeres. Resultados similares a los de Espelague y cols..$^{22}$ donde se estableció que escolares involucrados en bullying presentan mayor riesgo de conducta e ideación suicida respecto a no involucradas, teniendo las niñas mayor riesgo, incluso después de corregir por depresión.

\section{Estudios longitudinales}

Klomek y cols. ${ }^{21}$ demostraron que adolescentes hospitalizados por intento suicida no involucrados en bullying tuvieron al inicio del estudio menores niveles de depresión comparado con el grupo involucrado como perpetrador y victima simultáneamente (media $=18,5$ vs 24,8 respectivamente, $\mathrm{p}<0,01$ ), diferencia que no se mantuvo en el seguimiento a 2 años.

El estudio longitudinal de Undheim y cols. ${ }^{23}$ evaluó síntomas depresivos e ideación suicida en dos tiempos distanciados por 1 año $(\mathrm{T} 1=14$ años y T2 $=15$ años $)$ en una población adolescente. Las víctimas y perpetradores de bullying presentaron mayores puntajes en la escala de ideación suicida en T1 (media $=1,48$ y 1,13, respectivamente) respecto de los no involucrados en bullying (media $=0,45)$. Los niveles de suicidio más altos sólo se mantienen en las víctimas en la evaluación en T2 (media $=1,31$ vs $0,59, \mathrm{p}<0,001)$. El análisis multivariado evidenció que predecían ideación suicida factores como el haber sido víctima de bullying, presentar puntajes altos en MFQ (Mood and Feelings Questionnaire) y tener conducta agresiva. El análisis de interacción bidireccional mostró para ambos sexos un efecto significativo entre bullying en T1 y depresión en T2 $\left(\mathrm{R}^{2}=36.2 \%, \mathrm{p}<0,001\right)$. El análisis multivariado longitudinal mostró que haber sido víctima de $b u$ llying en T1 $(\mathrm{p}<0,001)$ y puntaje alto en el MFQ en T2 $(\mathrm{p}<0,001)$ predecía ideación suicida en T2.

Respecto a población sudamericana, sólo se encontró un estudio de Sandoval-Ato y cols. ${ }^{24}$, que incluyó 289 escolares de la ciudad de Piura, Perú. Por medio de análisis multivariado se identificó que incrementó el riesgo suicida tanto el presentar puntaje en tercio superior en la escala de evaluación de bullying $(\mathrm{RPa}=1,83$, $\mathrm{p}=0,015)$ como el estar deprimido $(\mathrm{RPa}=3,32$, $\mathrm{p}<0,001)$.

\section{d) Relación entre bullying, cyberbullying, depresión y suicidio}

Tres estudios transversales consideraron la forma cibernética del bullying, diferenciándola de su forma tradicional, encontrándose en ambas formas resultados similares en su relación con depresión y suicidio.
En Williams y cols. ${ }^{25}$ se determinó tras análisis bi-variado que el ser víctima de bullying verbal/social predijo síntomas depresivos tanto para hombres $(\mathrm{OR}=0.079, \mathrm{p}<0,05)$ como para mujeres $(\mathrm{OR}=0,25$, $\mathrm{p}<0,05)$, mientras que ser víctima de cyberbullying se asoció con síntomas depresivos ( $\mathrm{OR}=0,139, \mathrm{p}<0,05)$, ideación suicida $(\mathrm{OR}=0,168 \mathrm{p}<0,001)$ e intentos suicidas $(\mathrm{OR}=0,091, \mathrm{p}<0,01)$ sólo en las mujeres.

El artículo de Bonanno y cols. ${ }^{26}$ determinó que, con excepción del perpetrar bullying físico, las formas de perpetración o victimización física, social, verbal y cibernética estuvieron correlacionadas significativamente con depresión. Por modelos de regresión se observó que el antecedente de ser víctima de bullying social, verbal y cibernético tienen efecto significativo en síntomas depresivos, mientras que el ser víctima de bullying físico, no lo tiene. También se estableció que el perpetrar bullying verbal y cibernético tuvo impacto significativo en sintomatología depresiva, mientras que las formas física y social no.

El artículo de Bauman y cols. ${ }^{19}$ determinó que el ser víctima de bullying fue predictor de depresión tanto para hombres $(\beta=0,20, \mathrm{p}<0,001)$ como mujeres $(\beta=0,13, \mathrm{p}<0,01)$, mientras que ser víctima de $c y$ berbullying fue predictor de depresión sólo en mujeres ( $\beta=0.24, p<0,001)$. También determinaron que el perpetrar bullying en su forma tradicional fue predictor de depresión sólo en mujeres $(\beta=0,11, \mathrm{p}<0,05)$ y que el perpetrar cyberbullying no fue predictor de depresión en hombres ni mujeres. En el análisis de mediación determinaron que la depresión es un mediador entre ser víctima de bullying tradicional e intento de suicidio tanto para hombres como para mujeres, mientras que ser víctima de cyberbullying e intento de suicido fue mediado por depresión sólo en mujeres. Por otra parte, el perpetrar bullying en su forma tradicional y presentar intento de suicidio fue mediado por depresión sólo en mujeres. El perpetrar cyberbullying y presentar intento de suicidio no fue mediado por depresión.

\section{Discusión}

Los resultados de esta revisión ponen de manifiesto la relación entre exposición a bullying y desarrollo de síntomas depresivos ${ }^{9,10-12,14,15}$. Esta correlación entre exposición a bullying y desarrollo de síntomas depresivos fue mayor en víctimas, aunque perpetradores y perpetradores-víctimas también desarrollan más sintomatología depresiva cuando se compara contra grupos no expuestos a bullying ${ }^{15}$. Lo antes mencionado plantea el desafío de identificar las experiencias de bullying durante una ventana temporal relativamente acotada, mediante la pesquisa de síntomas depresivos, 
y refuerza el bullying como un factor asociado a episodios depresivos en escolares. Pese a que uno de los estudios no encontró evidencia robusta que sostenga una relación entre bullying y depresión, el tamaño de la muestra $(n=206)$ era relativamente pequeño comparado con otros estudios ${ }^{13}$.

Si bien se logró establecer una asociación entre la participación en bullying e ideación suicida en adolescentes, es importante considerar que el bullying, por sí sólo, no parece explicar totalmente la aparición de ideación suicida o intentos de suicidio, sino que su efecto se potenciaría con el de otros factores de riesgo biopsicosociales concomitantes, siendo la ideación suicida un desenlace de origen multifactorial ${ }^{18}$. De esa forma, la simplificación de la afirmación de que el antecedente de bullying es equivalente al suicidio adolescente, no parece certera ${ }^{27}$, debiéndose explorar otros factores, en particular la presencia de depresión, factor claramente asociado a suicidio adolescente.

Por su parte, los datos disponibles hasta ahora muestran que una asociación entre cyberbullying, el desarrollo de síntomas depresivos e ideación o intentos de suicidio es significativa en mujeres ${ }^{25}$. También se estableció evidencia estadística para señalar que la depresión es un mediador entre exposición a cyberbullying e intento de suicidio en mujeres ${ }^{19}$.

Estos datos plantean la necesidad de ampliar la pesquisa del bullying más allá de los ámbitos escolares, hacia un espacio que es difícil de supervisar y controlar como lo es la internet y las redes sociales, entendiendo el cyberbullying como un determinante importante en la sintomatología depresiva y suicida.

Dentro de las limitaciones de los estudios revisados se encuentra la diversidad de instrumentos de medición utilizados para evaluar el síntoma mental (vivencia de bullying, depresión, ideación suicida). Del mismo modo se encontró una gran diversidad de tamaños muestrales entre los diferentes trabajos analizados, los que varían desde decenas (un artículo), centenas (ocho artículos), miles (ocho artículos), incluso llegando a decenas de miles (un artículo). Además, es necesario tener en cuenta que varios de los estudios están limitados a población adolescente con asistencia activa a establecimientos educacionales. Por lo tanto, habría un grupo de estudiantes de alto riesgo no representados en las muestras.

Es preciso mencionar que este artículo se limitó a evaluar la relación de bullying con depresión y suicidio, por lo que la literatura que relaciona el bullying con otras variables potencialmente relevantes (síntomas ansiosos, entre otros) no se consideró en esta revisión, impidiendo de esta manera un análisis que relacione el bullying con el desarrollo de otras patologías de salud mental.

\section{Conclusiones}

Pese a la gran variabilidad de tamaños de muestra e instrumentos utilizados en los estudios revisados, la evidencia establecería una relación significativa entre la exposición a bullying y desarrollo de depresión e ideación suicida en adolescentes, además de confirmar la mediación de los síntomas depresivos entre la exposición a bullying y suicidabilidad.

El bullying podría ser un factor de riesgo modificable en la incidencia de depresión e ideación suicida. Nuestra capacidad de predecir la inminencia del suicidio es aún muy baja ${ }^{28}$, por lo que es fundamental reafirmar los esfuerzos en la detección y prevención del bullying, ampliando su pesquisa a nuevos espacios de interacción social como lo son las redes sociales.

Dado que el bullying se asocia con frecuencia a problemas en el ámbito familiar y escolar, y para el desarrollo de psicopatología, es necesario abordar el problema desde un modelo de intervención integral, potenciando medidas de manejo y prevención tales como las iniciadas por la Ley de Violencia Escolar ${ }^{29}$ y las indicadas en el Programa Nacional de Prevención del Suicidio (PNPS) ${ }^{30}$, que hace esfuerzos por incluir el manejo de crisis, y prevención de la intimidación o violencia en el centros educativos.

La escasa literatura del tema en Latinoamérica, y la ausencia de ésta en Chile, plantea como necesario realizar estudios que permitan un mejor diagnóstico, prevención y manejo del bullying en nuestro país.

\section{Conflicto de intereses}

Los autores declaran no tener conflicto de intereses.

\section{Agradecimientos}

Comisión Nacional de Investigación Científica y Tecnológica de Chile (Conicyt), a través de Fondo Nacional de Desarrollo Científico y Tecnológico, Fondecyt Regular No 1171313 y CONICYT PIA/Anillo de Investigación en Ciencia y Tecnología ACT172121. 


\section{Referencias}

1. Romero M. Gobierno lamenta fallecimiento de estudiante del Nido de Águilas: "No es aceptable el bullying" [Internet]. Emol. 2018 [citado el 19 de marzo de 2019]. Disponible en: https://www.emol.com/noticias/ Nacional/2018/05/23/907198/Gobiernolamenta-fallecimiento-de-estudiantedel-Nido-de-Aguilas-No-es-aceptable-elbullying.html

2. Toro I. Bullying escolar 2018: 2.700 denuncias de acoso físico y psicológico se recibieron hasta septiembre [Internet]. La Tercera. 2019 [citado el 19 de marzo de 2019]. Disponible en: https://www. latercera.com/la-tercera-pm/noticia/ bullying-escolar-2018-2-700-denunciasde-acoso-fisico-y-psicologico-serecibieron-hasta-septiembre/495869

3. Olweus D. School Bullying: Development and Some Important Challenges. Annu Rev Clin Psychol. 2013;9(1):751-80.

4. Smith P, Cowie H, Olafsson R, Liefooghe A. Definitions of Bullying: A Comparison of Terms Used, and Age and Gender Differences, in a Fourteen-Country International Comparison. Child Dev. 2002;73(4):1119-33.

5. Encuesta Nacional de Violencia en el Ámbito Escolar (ENVAE) [Internet]. Seguridadpublica.gov.cl. 2014 [citado el 10 de julio de 2019]. Disponible en: http://www.seguridadpublica.gov.cl/ media/2016/11/Presentaci\%C3\%B3nENVAE.pdf

6. OECD. Health status - Suicide rates OECD Data [Internet]. OECD. 2017 [citado el 10 de julio 2019]. Disponible en: https://data.oecd.org/healthstat/ suicide-rates.htm

7. OECD. Suicides [Internet]. OECD iLibrary. 2016 [citado el 10 de julio de 2019]. Disponible en: https://read. oecd-ilibrary.org/economics/oecdfactbook-2015-2016/suicides_factbook2015-88-en\#page 1

8. Silva D, Vicente B, Saldivia S, Kohn R. Conducta suicida y trastornos psiquiátricos en Chile, un estudio poblacional. Rev Med Chile. 2013;141(10):1275-82.

9. Ford R, King T, Priest N, Kavanagh A. Bullying and mental health and suicidal behaviour among 14- to 15-year-olds in a representative sample of Australian children. Aust N Z J Psychiatry. 2017;51(9):897-908.

10. Lereya S, Copeland W, Costello E, Wolke D. Adult mental health consequences of peer bullying and maltreatment in childhood: two cohorts in two countries. Lancet Psychiatry. 2015;2(6):524-31.

11. Lardier D., Barrios V., Garcia-Reid P., Reid R. Suicidal ideation among suburban adolescents: The influence of school bullying and other mediating risk factors. J Child Adolesc Ment Health. 2016;28(3):213-31.

12. Jones H, Bilge-Johnson S, Rabinovitch A, Fishel H. Self-reported peer victimization and suicidal ideation in adolescent psychiatric inpatients: the mediating role of negative self-esteem. Clin Child Psychol Psychiatry. 2014;19(4):606-16.

13. Kerr D, Gini G, Capaldi D. Young men's suicidal behavior, depression, crime, and substance use risks linked to childhood teasing. Child Abuse Negl. 2017;67:32-43.

14. Klomek A, Kleinman M, Altschuler E, Marrocco F, Amakawa L, Gould M. High school bullying as a risk for later depression and suicidality. Suicide Life Threat Behav. 2011;41(5):501-16.

15. Le H, Nguyen H, Campbell M, Gatton M, Tran N, Dunne M. Longitudinal associations between bullying and mental health among adolescents in Vietnam. Int J Public Health. 2017;62(Suppl 1):51-61.

16. King C, Horwitz A, Berona J, Jiang Q. Acutely suicidal adolescents who engage in bullying behavior: 1-year trajectories. J Adolesc Health. 2013;53(1 Suppl):S43-50.

17. Feng C, Waldner C, Cushon J, Davy $\mathrm{K}$, Neudorf C. Suicidal ideation in a community-based sample of elementary school children: A multilevel and spatial analysis. Can J Public Health. 2016;107(1):e100-5.

18. Hong L, Guo L, Wu H, et al. Bullying, Depression, and Suicidal Ideation Among Adolescents in the Fujian Province of China. Medicine (Baltimore). 2016;95(5):e2530.

19. Bauman S, Toomey R, Walker J. Associations among bullying, cyberbullying, and suicide in high school students. J Adolesc. 2013;36(2):341-50.

20. Meltzer H, Vostanis P, Ford T, Bebbington P, Dennis M. Victims of bullying in childhood and suicide attempts in adulthood. Eur Psychiatry. 2011;26(8):498-503.

21. Klomek A, Kleinman M, Altschuler E, Marrocco F, Amakawa L, Gould M. Suicidal adolescents' experiences with bullying perpetration and victimization during high school as risk factors for later depression and suicidality. J Adolesc Health. 2013;53(1 Suppl):S37-42.

22. Espelage D, Holt M. Suicidal Ideation and School Bullying Experiences After Controlling for Depression and Delinquency. J Adolesc Health. 2013;53(1):S27-31.

23. Undheim A, Sund A. Involvement in bullying as predictor of suicidal ideation among 12- to 15-year-old Norwegian adolescents. Eur Child Adolesc Psychiatry. 2013;22(6):357-65.

24. Sandoval R, Vilela M, Mejia C, Caballero J. [Suicide risk associated with bullying and depression in high school]. Rev Chil Pediatr. 2018;89(2):208-15.

25. Williams $S$, Langhinrichsen-Rohling $J$, Wornell C, Finnegan H. Adolescents Transitioning to High School: Sex Differences in Bullying Victimization Associated With Depressive Symptoms, Suicide Ideation, and Suicide Attempts. J Sch Nurs. 2017;33(6):467-79.

26. Bonanno R, Hymel S. Cyber bullying and internalizing difficulties: above and beyond the impact of traditional forms of bullying. J Youth Adolesc. 2013;42(5):685-97.

27. Toro I, Leiva L. Suicidio: 13 escolares se quitaron la vida en Santiago en 2018 y es la segunda causa de muerte en adolescentes en el país [Internet]. La Tercera. 2019 [citado el 19 de marzo de 2019]. Disponible en: https://www. latercera.com/la-tercera-pm/noticia/ suicidio-13-escolares-se-quitaron-la-vidaen-santiago-en-2018-y-es-la-segundacausa-de-muerte-en-adolescentes-en-elpais/494216.

28. Appleby L, Shaw J, Amos T, et al. Suicide within 12 months of contact with mental health services: national clinical survey. BMJ. 1999;318(7193):1235-9.

29. Ley $\mathrm{N}^{\circ}$ 20.536: Sobre Violencia Escolar. Santiago, Chile: Ministerio de Educación; 2011.

30. Ministerio de Salud. Programa nacional de prevención del suicidio: Orientaciones para su implementación. MINSAL; 2013. 